\title{
AIAA 99-0776
}

Automatic Generation of CFD-Ready Surface Triangulations from CAD Geometry

M. J. Aftosmis, M. Delanaye

NASA Ames Research Center,

Moffett Field, CA

R. Haimes

Massachusetts Institute of Technology

Cambridge, MA

\section{7th AIAA Aerospace Sciences Meeting and Exhibit January 11-14, 1997}




\title{
Automatic Generation of CFD-Ready Surface Triangulations from CAD Geometry
}

\author{
M. J. Aftosmis \\ NASA Ames Research Center \\ Moffett Field CA 94035 \\ M. Delanaye \\ NASA Ames Research Center \\ Moffett Field CA 94035 \\ R. Haimes ${ }^{¥}$ \\ Massachusetts Institute of Technology
}

\begin{abstract}
This paper presents an approach for the generation of closed manifold surface triangulations from CAD geometry. CAD parts and assemblies are used in their native format, without translation, and a part's native geometry engine is accessed through a modeler-independent application programming interface (API). In seeking a robust and fully automated procedure, the algorithm is based on a new physical space manifold triangulation technique which was developed to avoid robustness issues associated with poorly conditioned mappings. In addition, this approach avoids the usual ambiguities associated with floating-point predicate evaluation on constructed coordinate geometry in a mapped space. The technique is incremental, so that each new site improves the triangulation by some well defined quality measure. Sites are inserted using a variety of priority queues to ensure that new insertions will address the worst triangles first. As a result of this strategy, the algorithm will return its "best" mesh for a given (prespecified) number of sites. Alternatively, the algorithm may be allowed to terminate naturally after achieving a prespecified measure of mesh quality. The resulting triangulations are "CFD-ready" in that: (1) Edges match the underlying part model to within a specified tolerance. (2) Triangles on disjoint surfaces in close proximity have matching length-scales. (3) The algorithm produces a triangulation such that no angle is less than a given angle bound, $\alpha$, or greater than $\pi-2 \alpha$. This result also sets bounds on the maximum vertex degree, triangle aspect-ratio and maximum stretching rate for the triangulation. In addition to the output triangulations for a variety of CAD parts, the discussion presents related theoretical results which assert the existence of such an angle bound, and demonstrate that maximum bounds of between $25^{\circ}$ and $30^{\circ}$ may be achieved in practice.
\end{abstract}

\section{Introduction}

Mesh generation has long been recognized as a bottleneck in the CFD process. ${ }^{[1]}$ The last decade has witnessed a myriad of international and domestic conferences and symposiums aimed at focusing research on this impediment. Unstructured, hybrid, and Cartesian mesh methods are all aimed at simplifying the mesh generation task for complex configurations. The success of these approaches is well represented in the literature and with an appropriate initial surface triangulation. the volume mesh generation can generally be accomplished in a relatively automated fashion in minutes-to-hours on an engineering workstation. ${ }^{[2-7]}$ As faster processors, with better access to memory, continue to shrink the wall-clock time

Research Scientist, Senior Member AlAA.

$\ddagger$ NRC Associate, Member AIAA.

$¥$ Principal Research Engineer, Member AIAA.

This paper is declared a work of the U.S. Government and is not subject ot copyright protection in the United States. required for both mesh generation and flow solution, the man-hour intensive task of generating an initial surface triangulation from a CAD geometry promises to become an ever larger fraction of the bottleneck. Additionally, if the user must be involved in the extraction of surface data from CAD, then mesh adaptation - which involves enriching the discretization on the body surface - will remain an elusive goal.

Historically, surface discretization has been one of the least automated steps in the numerical simulation cycle, and for good reason. Due to its dependence on implicitly defined surfaces and curves, CAD data is by its nature imprecise. Various geometry engines typically demonstrate discrepancies in their interpretations of the same entities. As a result, "repair" of CAD surfaces has become an area of substantial research. ${ }^{[8][9]}$ This problem is exacerbated when CAD models are output in many of the standard formats, since such files frequently do not include important topological and construction information along with the entity geometry.

In addition to the vagaries of the data and modeling systems, many surface triangulation schemes treat surfaces individually. Thus, the length scale of the triangulation on a particular component may not be commensurate with that of other components which are in close proximity on the full configuration. In many interactive triangulation systems, it becomes the user's role to identify such situations and enforce length scale compatibility between nearby components.

In response to these and other requirements for user assistance, many in the research and industrial CFD communities have adopted an interactive paradigm for surface mesh generation. The commercial unstructured mesh generators in Refs. [7],[10] and [11] all interact with CAD data through files which have been translated from their CAD native environment to some standardized format (namely IGES ${ }^{[12]}$,

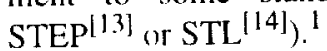

This paper adopts an alternative paradigm. The approach interfaces with the CAD system in a dynamic manner via calls to CAD native routines. By accessing the model in its native environment, this approach avoids translation to a format which can deplete the model of topological information. This is important since it avoids the consistency conflicts that

1 Recent releases of some of this software now supports "direct" interfaces which do read parts in their native formats, however, this practice is not the norm. 
can occur when two different geometry engines attempt to infer topological information from imprecise data.

To avoid placing CAD specific calls in the software, we access CAD function calls through an Application Programming Interface (API) known as CAPRI. ${ }^{[15]}$ This library presents a standardized interface to the application program for various CAD systems. ' CAPRI supports a variety of operations like truth testing, geometry construction, and entity queries.

Maintaining the consistency of the models by direct manipulation of CAD parts and assemblies is the first step that this work takes toward building a robust method for surface triangulation. A basic premise of this approach is that we resolve consistency problems on as simple a model as possible, and maintain this consistency as the triangulation evolves and becomes more complex.

\subsection{Abstract Geometric Structures}

Following the approach of Yap, ${ }^{[16]}$ our approach toward generating a robust geometric algorithm contends that a geometric structure, $D$, consists of four elements: ${ }^{2}$

$$
D=(G, \lambda, \Phi(\mathbf{z}), \mathbf{z})
$$

Where the graph, $G=(V, E)$ is a directed set of vertices, $V$, and edges, $E . \lambda$ is a function describing the index labels of the graph. $\Phi$ is a geometric operator which represents the consistency predicates for the connectivity and is a function of the actual coordinates $\mathbf{z}$. $G$ represents a tessellation of the vertices and is therefore purely combinatorial. A structure is said to be consistent if the predicate $\Phi(\mathbf{z})$ holds.

As an example, a 2-D Delaunay triangulation algorithm usually makes use of an inCircle predicate ${ }^{[17]}, \Phi_{\text {inCircle }}$ which establishes $G$ by insisting that the circumcircle of the triangle $\Delta_{a, b, c}$ can contain no other vertex in the graph. If such a predicate holds for every triangle in $G$, then this instance of the geometric structure $D$ is said to be consistent.

\subsection{Robustness}

Consistent CAD Models

This interpretation offers direct insights into the formulation of a robust algorithm for creating triangulations of CAD volumes. The rational B-splines used to describe surfaces in most CAD systems are implicitly defined for physical space coordinates of the geometry. Therefore, the constructors for vertex geometry generally require a Newton solve carried 10 some internal tolerance. Since the results of this construction will be subject to both tolerance and round-off error, the system may then "nudge" the constructed point, $z_{i}$, to some nearby exactly representable location (on an integer grid, for example). If the geometry engine's predicate for determining if $z_{i}$ is on some surface, $S$ is $\underset{\text { onsurf }}{\Phi}\left(z_{i}, S\right)$, is consistent then it

1. CAPRI currently supports ProEngineer ${ }^{\mathrm{TM}}$ ParaSolids $^{\mathrm{TM}}$ and IDEAS ${ }^{\mathrm{TM}}$ with CATIA ${ }^{\mathrm{TM}}$ support in beta test.

2. Ref.[16] actually writes eq.(1) as $D=(G, \lambda, \Phi(\mathbf{z}), I)$ where $l$ is a mapping from the input parameters $\mathbf{c}$ to $\mathbf{z}, I: \mathbf{z} \rightarrow \mathbf{c}=\left(c_{1} \ldots r_{n}\right) \in \mathfrak{g i}^{d}$. will return "true" when later queried if $z_{j}$ lies on the surface. However, if $\tilde{\Phi}_{\text {onsurf }}$ now represents some user-defined predicate which may be ignorant of the systems construction rules, then it is very unlikely to return consistent results.

The CAPRI API allows us to access a subset of the CAD geometry engine's constructors, queries and predicates in order for the algorithm in our application to maintain a consistent representation of the model. Implementationally, we adopt a multi-threaded programming approach which starts the geometry engine on its own thread in order to respond to queries from the main triangulation thread.

\section{Physical Space Triangulation}

A variety of existing surface meshing techniques adopt a mapped-space approach for generating surface triangulations. In this approach, the surface and its bounding curves are triangulated in a 2-D parameter space, which may or may not have some additional scaling imposed. Ref.[18] provides a mathematical description of the Non-Uniform Rational BSpline (NURBS) surfaces typically used in CAD systems. Here we note only that an iterative method is required to solve for the physical space coordinates of a position specified on the surface in the parameter space. This process involves division of two (generally) high-order polynomials, and it therefore incurs both floating-point round-off error and error associated with tolerancing for the convergence of the itcrative solve. As a result, computed coordinates in the mapped space are necessarily noisy and cannot be considered exact values. Two consequences of this approach are:

1. Since the error bounds on the input are unknown, evaluation of the triangulation predicate (e.g. $\tilde{\Phi}_{\text {inCircle }}$ ) are unlikely to robustly produce consistent results (see Fig. 19 Ref.[17], also [19] and [201).

2. The polynomial basis for the NURB may be high-order, and therefore small errors in parameter space may produce dramatic results in physical space - even within the subspace for which the surface is defined. The likelihood of encountering poorly conditioned mappings is the primary reason that $C A D$ repair software generally attempts to re-normalize the NURBS surface and recast it using basis polynomials with as low an order as possiblc $^{[8]}$.

These two observations have led the current work to focus on physical space triangulation techniques. In this approach, we construct a manifold triangulation on the surface, and evaluate the triangulation predicates in $\Re^{3}$. New sites are constructed by the CAD geometry engine and, since this output is consistent with the system's internal predicates, it is considered exact by the external predicates of the triangulation algorithm. The presentation in $\$ 3$ places emphasis on minimizing the floating-point error in predicate evaluation and notes the computational requirements needed to make this evaluation exact.

\section{The CAPRI API}

Our basic approach is to take a crude manifold triangulation 

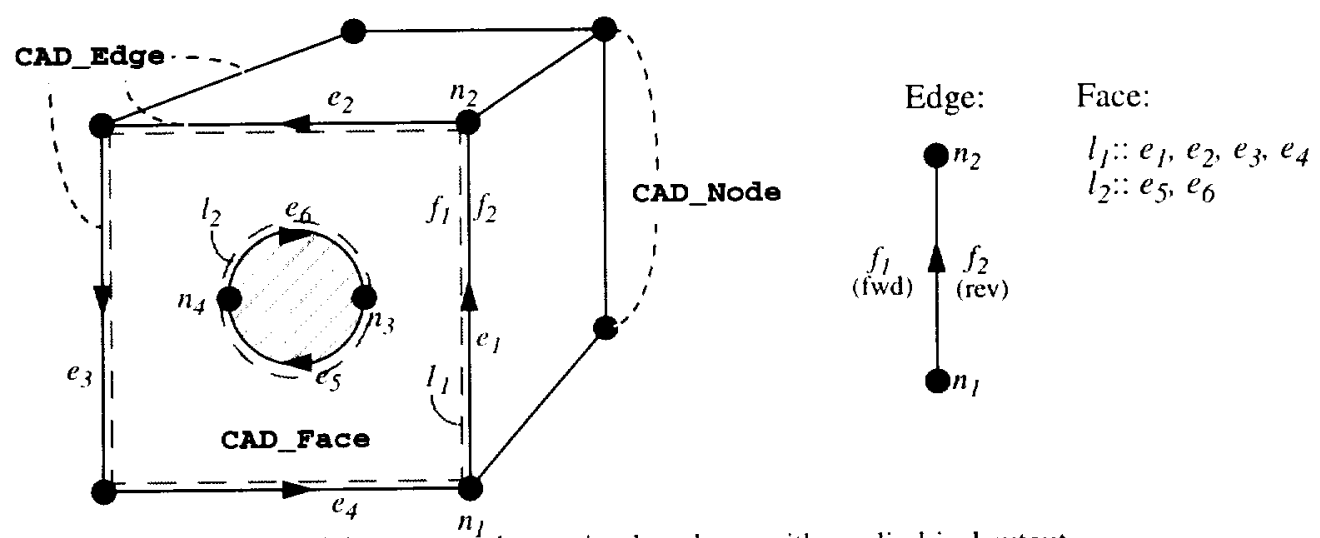

Figure 1. CAPRI data structured demonstrated on a simple volume with a cylindrical cutout.

of each closed volume in the CAD assembly, and improve it until it satisfies a preset measure of mesh quality, or produces a preset number of triangles. A variety of mesh quality measures may be defined within this framework, and this preliminary investigation focuses on three such criteria. (1) The mesh must be free from small angles (sliver triangles). (2) Edges in the triangulation must not deviate from the underlying model by more than a prescribed tolerance. (3) Length scales on nearby (possibly disjoint) surfaces must be commensurate

\subsection{CAPRI Volumes}

As discussed in the introduction, CAD entities are accessed through the CAPRI programming interface. ${ }^{[15]}$ CAPRI provides a layer of indirection such that $C A D$ system specific data may be accessed by an application program using CAD system neutral function calls. Figure 1 presents an abstract view of the entities that CAPRI provides. A cad_node is the lowest dimensional entity and corresponds to a point in 3space. A cad_edge has a cad_node at both ends. Each is directed from its origin, $O$, to its destination, $D$. Cad_edges are not assumed to be simplicial and may follow a general curve in space (see $e_{5}$ and $e_{6}$ in Fig. 1). Each edge is connected to two cad face entities. In general, these faces are composed of several loops and are not assumed planar, since they follow the underlying parameterization of the surface. A cad_face is composed of one or many loops, which are collections of oriented edges. Loops are oriented such that the surface of the cad_face lies inside them when they are tra-

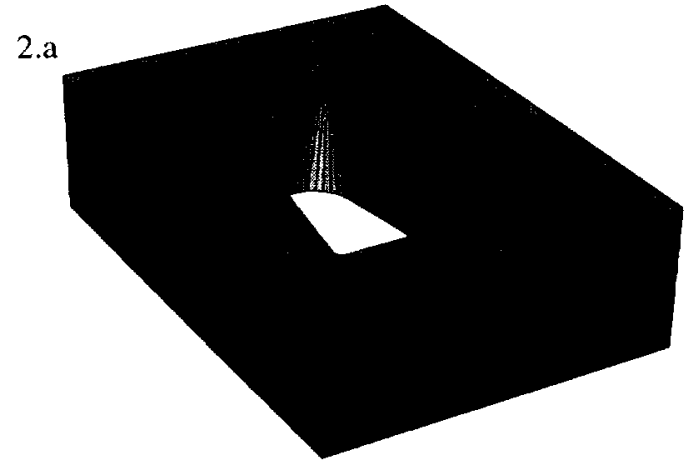

versed in a counterclockwise circuit when viewed from a point outside the solid. This convention permits holes in a surface to be described by a clockwise loop. In the figure, cad_face, $f_{l}$, consists of loops $l_{l}$ and $l_{2}$, each of which is composed of cad_edge entities. The edge ordering of $l_{2}$ indicates that it is clockwise, and therefore describes a hole in $f_{l}$. Edges and faces have an underlying parameterization, and while points may be queried for their parametric values $(u, v)$, details of this ruling are not exposed to the application program.

\subsection{Initial Manifold Triangulation}

A central theme in the present approach is the maintenance of a closed volume throughout the procedure. CAPRI returns a simplicial decomposition of each of the $m$ cad_face entities, $S_{i}$, where $S_{i} \in\left\{S_{1}, S_{2}, \ldots, S_{m}\right\}$. Each of these triangulations are manifold within their respective cad_edges. In addition, an indexing function, $\lambda_{C}$, is returned for each cad_volume. Therefore a simplicial, manifold representation of each cad_volume, $S_{C}$ may be constructed by taking the union of the decompositions of all the cad_face entities of a volume, subject to the indexing $\lambda_{C}$.

$$
S_{C}=\cup_{i} S_{i} \quad \forall i \in\{1, \ldots, m\}
$$

Figure 2 displays an example of this initial triangulation for a simple part. The manufacturing die shown has 14 cad_face entities and the initial triangulation, $S_{C}$, has 270 triangles. As is typical, this triangulation is quite irregular, and planar regions are decomposed into as few triangles as possible.

Figure 2. Initial closed, manifold, surface triangulation, $S_{6}$, of a CAD model for a manufacturing die. Underlying CAD entities exposed to application program are labeled in the frame on the right. 
Extremely high aspect ratio triangles are also common in such boundary triangulations. Figure $2 . \mathrm{b}$ labels selected CAD entities on this triangulation. Notice that although some cad_face sites may be present, this initial triangulation is essentially a boundary triangulation and the number of triangles is proportional to the number of cad_edges.

Despite the poor quality of the triangulation, the structure in Fig.2 has several desirable properties. Namely, it is manifold, oriented and closed. We wish to improve this triangulation by adding sites on both the cad_edge and cad_face entities and by enforcing an external predicate governing the type of triangulation.

\section{Mesh Improvement}

Our approach for manifold surface triangulation traces its roots to work on quality triangulations of Planar Straight Line Graphs (PSLGs) ${ }^{[21][22][23]}$ and related work on quality triangulations of manifold surfaces. ${ }^{[24]}$ Work in this field began with the efforts of Ref.[21] which presented an algorithm with both shape and size guarantees. The resulting meshes were size-optimal and had no triangle with an aspect ratio greater than 5 . In this context, the aspect ratio $A R$, of a triangle, is defined as the length of the longest edge divided by that of the shortest one. One can show that if $\alpha$ is the smallest angle of a triangle, then

$$
\frac{1}{|\sin \alpha|} \leq A R \leq \frac{2}{|\sin \alpha|} .
$$

Therefore $\alpha$ is frequently used to describe the quality of a given triangle.

Before presenting the manifold triangulation technique, this section first recounts a related algorithm for quality triangulation for PSLGs from Ref.[23]. It then present a fundamentally similar algorithm for triangulating curved surfaces and note which aspects of the PLSG method have been relaxed in the extension.

\subsection{Quality Triangulation of PSLGs}

While the manifold surface triangulation technique of Ruppert $^{[24]}$ and the PSLG method of Chew ${ }^{[23]}$ are similar in many respects, our manifold technique follows Ruppert's approach more closely. Section 3.2 addresses some of the reasoning behind this choice.

An essential feature of the algorithm is the notion of an encroached constraining edge. As illustrated in Figure 3, a constraining edge, $\overline{O D}$, is said to be encroached upon if any other site (visible to $\overline{O D}$ ) lies within the diametral circle of the edge.

If one recalls that the circumcenter of a right triangle falls on the hypotenuse, then its easy to show that for a triangulation which is Delaunay or locally maxmin, a predicate for encroachment may be formulated as a vector dot product. Thus for similarly sized ${ }^{1}$ floating-point data with $p$-bit significands, this predicate can be computed exactly in a register with a $2 p$-bit significand ${ }^{[20]}$. In a practical sense, this implies that as long as the edges are small with respect to

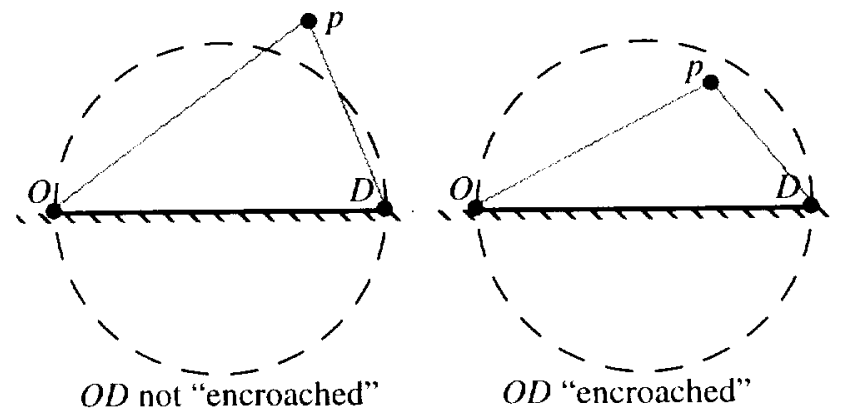

Figure 3. Constraining edge $\overline{O D}$ and its diametral circle. The edge is "encroached" if any site, $p$, falls within the diametral circle of $O D$

their distance from the origin, this predicate will be exact if computed in double-precision, using single-precision data.

In the algorithms, $\tilde{\Phi}_{\text {Encrouched }}(e)$ denotes application of this predicate $t o$ an edge, $e$. The ( $)$ superscript is used to remind us that since this predicate is part of our triangulation algorithm, it is not native to the CAD system.

The presentation of Ref.[23] recovers the constraining edges of the triangulation as the algorithm advances. To clarify its relation with our manifold triangulation technique, we recast the original algorithm assuming that it begins with a constrained boundary triangulation of the input vertices, $V$, of the constraint edges. Furthermore, this initial triangulation is assumed to be the constrained Delaunay triangulation of the input sites, $C D T(V)$.

The algorithm is quite elegant in that it consists of only two major operations:

1. Split a constrained edge: Add a site to the mid-point of a constraining edge, and replace the original edge with the two new edges in the constraint list.

2. Split a triangle: Add a site to the circumcenter of a triangle, $t$. Note that if the triangle is obtuse, this site will not fall within $t$.

\section{Algorithm Q: Quality triangulation of a PLSG}

Input: Planar Straight Line Graph, $X$, with input vertices, $V_{i n}$. Target angle, $\alpha$.

Output: $\quad C D T\left(V_{u u t}\right)$ with all angles $\geq \alpha$.

Initialize: Compute $C D T\left(V_{i n}\right)$. Build minimum angle priority queue, $P Q_{\text {min }}$ with $t_{P Q}$ denoting triangle at head of queue, having min angle $\theta_{P Q}$

1. Apply $\tilde{\Phi}$ Encroached to all constraint edges:

While (any constraining edge is encroached) ) Split constrained edge. Update $C D M V)$, Update $P Q_{\text {min }}$.

2. While $\left(\theta_{P Q}<\alpha\right)$ t

2.a Let $p$ be the circumcenter of $t_{P O}$.

2.b If ( $p$ encroaches any constraining edge, $e$ )

2.c Split constrained edge. Update $C D T(V)$, Update $P Q_{\min }$.

2.d Else Split triangle $t_{P Q}$ : Add $p$ to $V$. Update $C D T V)$, Update $P Q_{\text {min }}$.

3. Output $C D M(V)$.

1. The qualifier "similarly sized" is necessary to guard against the case where a coordinates of one point is less than half that of another. Extended precision would be required in such a case. 
While simple, Ref.[23] proves that Alg.Q produces triangulations with the following desirable properties.

1. Quality: An angle bound $20.7^{\circ}$ is guaranteed, and values as high as $30^{\circ}$ may be achieved in practice.

2. Output Size: The size of the output triangulation is within a constant of the optimal number of triangles required to satisfy the angle criteria.

3. Size Optimality: Small input constraint edges are surrounded by proportionally small triangles. Nearby triangles have similar sizes, and the size variation of triangles in the mesh is proportional to the distance between them.

\subsection{Difficulties on Curved Surfaces}

In Ref.[24], Chew presents a quality triangulation technique which is closely related to Alg.Q. This work raises a number of difficulties associated with the extension of the PSLG method to curved surfaces.

Alg.Q has two salient aspects. (1) The triangulation is constrained Delaunay. (2) New sites are added at circumcenters. In making these observations, Chew notes that a straightforward definition of a circle on a surface is the loci of points on the surface which are equidistant from another point on the surface, where all distances are measured using the geodesic distance along the surface. While straightforward, this definition is problematic. Distances along the surface must be measured in physical space, and will therefore be expensive to compute on NURBS surfaces. In addition, due to the inherent error in finding the coordinates of a point on such a surface, robust predicates based upon this definition will be difficult to formulate. Finally, Chew notes that this definition has less subtle and non-intuitive consequences. A circle whose center lies near the base of a sharp spire, for example, may reach completely around the spire without also including the tip of the spire.

To circumvent such difficulties, the method in [24] makes use of an alternative definition of a circle. In the plane, the three vertices of a triangle define a unique circle. In 3-dimensions, however, an infinite family of spheres may be passed through those three points. Connecting the line through the centers of this family of spheres and intersecting this line with the surface identifies a particular sphere in this family. The circumcenter of the triangle may be defined to be the loci of points at the intersection of this particular sphere and the surface. Once this sphere is found, then the $\tilde{\Phi}_{\text {incircle }}$ triangulation predicate may be evaluated by simply computing distances in three dimensions as a vector magnitude.

Despite the effort, problems still exist with this predicate. (1) If the triangulation does not resolve the underlying surface closely enough, the line of circumsphere centers will not necessarily intersect the surface. Alternatively, it may also intersect the surface in multiple locations places. (2) Computing the intersection of this line with the surface will require an iterative method and will therefore be computationally inten- sive. (3) Once this intersection is successfully located on the surface, one must determine which triangle the point falls inside. Since the triangulation only matches the surface at the vertices, ambiguous situations may arise when two triangles claim ownership of the same site (near a ridge, for example). (4) On a curved surface, the circumcenters of two triangles with a shared edge may not be consistent. Specifically, when $\tilde{\Phi}_{\text {in Circle }}$ tests one of the triangles against the opposite vertex of the other, the results may not agree when the roles of the triangles are reversed. Lemma 5 in Ref.[24] shows this situation will arise if the normal vectors of the triangles vary by more than $\pi / 2$. A successful algorithm must guard against $\tilde{\Phi}_{\text {incircle }}$ becoming inconsistent.

\subsection{A New Surface Meshing Algorithm}

These outstanding ambiguities and computational expense motivated a search for a more straightforward algorithm. Our method makes two fundamental changes.

- To avoid the ambiguity associated with the definition of $\tilde{\Phi}_{\text {in Circle, }}$ we do not attempt a Delaunay triangulation of the curved surface. Instead we seek a triangulation which is everywhere locally maxmin. While this may seem to constitute a dramatic relaxation, recall that the goal is a practical algorithm, and we have no reason to prefer strictly Delaunay output triangulations. In addition, since one property of a Delaunay triangulation is that it is maxmin, this choice is worth investigating.

- When all angles of a triangle are acute, the circumcenter falls within the triangle. Ownership of the new site can then be uniquely assigned to this triangle. However, when a triangle is obtuse, ownership can become less clear. Therefore we make a simple choice: When a triangle is obtuse, we insert the new site at the centroid of the other triangle sharing the edge opposite the obtuse angle. Figure 4. illustrates this insertion rule. If $t_{i}$ is an obtuse triangle, then the new sites are added at the centroid of $t_{o p p}$.

While admittedly ad-hoc, this site insertion strategy is not as arbitrary as it may initially seem. As a particular angle of $t_{i}$ opens up in the transition from acute to obtuse, the circumcenter will pass from within $t_{i}$ to within $t_{o p p}$. The centroid of $t_{\text {top }}$ may therefore be thought of as an approximate circumcenter of $t_{i}$. Denoting the radius of the circumcircle of $t_{i}$ as $R$,
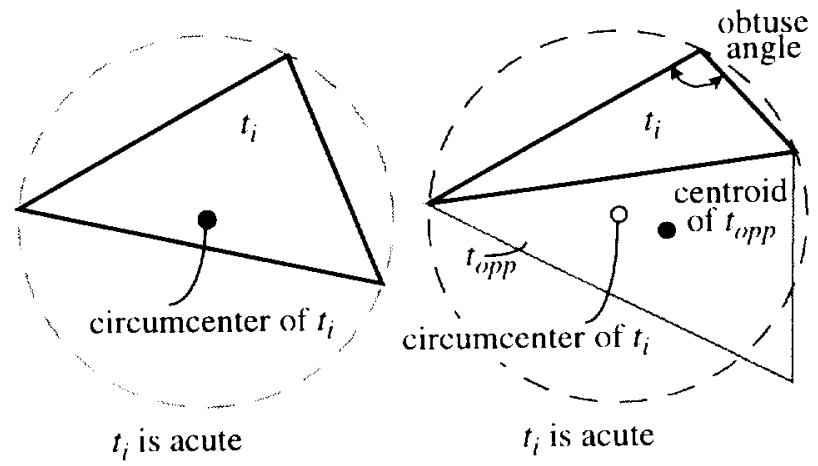

Figure 4. Sites are added at the centroid of a triangle if the triangle is acute. Otherwise they are added at the centroid of the triangle opposite the obtuse angle. 
it can be shown that if one chooses an approximate circumcenter within a distance $f R$ of the true circumcenter, then an angle bound of

$$
\alpha \leq \operatorname{asin}\left(\frac{1-f}{2}\right)
$$

may still be achieved. Ref. [24] contends that even using approximate circumcenters, angle bounds of $30^{\circ}$ may be achieved in practice.

With these changes, the manifold triangulation algorithm becomes:

\section{Algorithm M:}

\section{Quality Manifold triangulation of a CAD volume.}

Input: Underlying CAD volume, $P$, with initial triangulation $S_{C}$, and input vertices, $V_{i n}$.

Target angle, $\alpha$.

Output: $\quad \operatorname{CXN}\left(V_{\text {out }}\right)$ with all angles $\geq \alpha$.

Initialize: Compute constrained locally maxmin triangulation CXV $\left(V_{i n}\right)$, using cad_edge entities as constraints. Build minimum angle priority queue, $P Q_{m i n}$ with $t_{P Q}$ denoting triangle at head of queue, having min angle $\theta_{P Q}$

1. Apply $\bar{\Phi}$ Encroached to all constraint edges:

While (any constraining edge is encroached) \{ \} Split constrained edge. Update $C X \mathcal{N} V$, Update $P Q_{\min }$.

2. While $\left.\left(\theta_{P Q}<\alpha\right)\right\}$

2.a If ( $t_{P Q}$ is not obtuse)

Assign $t:=t_{P Q}$, with circumcenter $p$

Else Assign $\mathrm{t}:=t_{o p}$, with centroid $p$.

2 b If ( $p$ encroaches any constraining edge, $e$ )

2.c Split constrained edge. Update $C X \lambda(V)$, Update $P Q_{\text {min }}$.

2.d Else Split triangle $t$ :

3. Output $\operatorname{cxp}(V)$.

Add $p$ to $V$. Update $C X X(V)$. Update $P Q_{\text {min }}$

Note that when the algorithm terminates, it recovers the properties of quality and size-optimality cited after the presentation of Alg.Q in \$3.1. The bound on output size, however, depends on the site insertion strategy always inserting new vertices within the circumcircle of $t_{P Q}{ }^{[23]}$ and the modified strategy will not always guarantee this, thus the algorithm sacrifices strict proof of this property.

The new algorithm requires initialization with a transformation of the part's original constrained manifold triangulation $S_{C}$ to one which is everywhere locally maxmin. If we assume the existence of a triangulation predicate $\tilde{\Phi}_{X N}$ which can be enforced for every pair of triangles sharing an edge in the triangulation (similar to the application of $\tilde{\Phi}_{i n C i r c l e}$ ), then this initialization may be performed with edge sweeps followed by edge-swapping when a violation is encountered. While the possibility of multiple sweeps makes this a seemingly inefficient approach, we recall that the initial complexity of $S_{C}$ is only proportional to the number of cad_edges in the geometric structure. Thus this simplistic approach is not a problem.

Site insertion proceeds in a manner comparable to the PSLG algorithm. A convenient implementation makes use of the incremental insertion strategy of Ref.[25]. However, since the triangulation is no longer Delaunay, both forward and reverse propagation is necessary after edge swaps. Ref.[25] gives a modified point placement strategy for splitting encroached edges. Our implementation of Alg.M adopts this strategy without modification.

\subsection{Triangulation Predicates}

Algorithm $M$ rests on two new triangulation predicates. One test a triangle for an obtuse angle, $\bar{\Phi}_{o b}(t)$ and the other, $\tilde{\Phi}_{X N}(e)$, tests if the edge, $e$, shared by any two triangles maximizes the minimum angle in either triangle. This is accomplished by comparison with a swapped edge, $e$, which connects the opposite vertices of the two triangles. Our approach hinges on the hope of evaluating these predicates robustly in physical space.

Both of these predicates can be formulated with direct measurement of the angles in a mesh. Since a triangle has three points, it uniquely define a plane in three dimensions. Angle measurement in a plane is unambiguous - despite the fact that the triangles form a discrete manifold which is lower dimensional than the surrounding space. Numerically, we recall that since vertex geometry returned by the $\mathrm{CAD}$ engine is considered exact, and so the error bound on the input is identically zero.

$\tilde{\Phi}_{o b}(t)$ is applied by a logical or accumulation of $\bar{\phi}_{\angle j-1, j, j+1}$, where $j$ is a cyclic index running over the vertices of $t, j \in\{1,2,3\}$. This angle predicate is formed by comparison of the square of the edge opposite $j$ to that of a hypothetical edge formed by assuming that the edges of $t$ incident on $j$ form a right angle in the plane of $t$.

$$
\tilde{\Phi}_{o b}\left(t_{123}\right)=\tilde{\phi}_{o b}(\angle 123) \vee \tilde{\phi}_{o b}(\angle 231) \vee \tilde{\phi}_{o b}(\angle 312)
$$

where

$$
\tilde{\phi}_{L_{j-1, j, j+1}}= \begin{cases}T \text { if }\left|v_{j}-v_{j-1}\right|^{2}+\left|v_{j+1}-v_{j}\right|^{2}>\left|v_{j+1}-v_{j-1}\right|^{2} \\ F & \text { otherwise }\end{cases}
$$

Notice that Eq.(6) requires only subtraction and multiplication of data which is known exactly. Thus the computational requirements for exact evaluation are the same as for evaluation of the dot product for $\tilde{\Phi}_{\text {encroached }}$ in $\$ 3.1$.

Evaluation of $\tilde{\Phi}_{X N}(e)$ requires a more direct method of angle measurement. Figure 5 shows a construction for this measurement. Recalling that $\sin ()$ is monotone over the interval

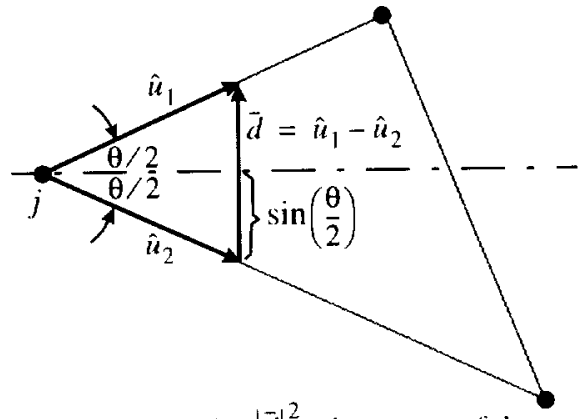

Figure 5. Construction for $|\bar{d}|^{2}$, the square of the magnitude of the difference of unit vectors incident on vertex $j$ 
$[0, \pi / 2]$ the construction in the figure shows that the difference of the unit vectors of the edges incident upon any vertex, $j$, is sufficient to define a vector $\bar{d}$ whose magnitude varies monotonically with the angle formed by the edges incident upon $j$. As in the preceding two predicates, computitionally, it is sufficient to evaluate only the square of this magnitude.

The computational requirements for this predicate are somewhat less simple. The computation of unit vectors requires robust computation of the inverse of a vector magnitude $1 /|\bar{V}|$. Thus, exact evaluation of $\tilde{\Phi}_{X N}(e)$ requires software arithmetic, like the packages in Refs.[26],[27] or [28]. Although this need must be viewed as a drawback, we note that it is confined to a single predicate, and to a relatively simple expression. Moreover, since the input geometry is exact, exact computation remains a feasible strategy. In the preliminary results shown in $\$ 4$, all computation was performed using only double-precision floating- point hardware, and the option of software arithmetic was not pursued.

\subsection{Edge Refinement}

Algorithm M drives small angles out of the evolving triangulation. In addition we wish to satisfy both chord-height and length-scale criterion. After initially creating a triangulation free of small angles we apply an edge-refinement procedure to enforce these two requirements. Since the algorithm is the same for either of these criterion, Algorithm E considers a generic edge-based scalar $\gamma(e)$. In our implementation chordheight is defined as the square of the distance from the middle of an edge to the corresponding location on the actual surface of the model (provided through CAPRI by the geometry engine).

Algorithm E: Edge Refinement of Manifold Triangulation.

Input: Underlying CAD volume, $P$, with current triangulation, $C X \mathcal{N}(V)$, and vertex set, $V$.

Edge criteria $\gamma$.

Output: $\quad C X \mathcal{N}\left(V_{o u t}\right)$ with all edges satisfying $\gamma(e)<\gamma$.

Initialize: Build priority queue, $P Q_{\gamma}$ with $e_{P Q}$ denoting edge at head of queue, having $\left.\gamma_{P} e_{Q}\right)$.

1. Apply $\tilde{\Phi}$ Encroached to all constraint edges:

While(any constraining edge is encroached)

J

Split constrained edge. Update $C X \mathcal{N}(\mathrm{V})$, Update $P Q_{\gamma}$

2. While $\left(\gamma\left(e_{P Q}\right)>\gamma\right)<$

2.a Let $p$ be midpoint of $e$.

2.b If ( $p$ encroaches any constraining edge, $e$ )

2.c Split constrained edge. Update $C X N(V)$, Update $P Q_{\gamma}$

2.d Else Split edge $e$ :

Add $p$ to $e$. Update $C X N(V)$, Update $P Q_{\gamma}$ \}

3. Output $\operatorname{CXN}(V)$.

Assuming that $\gamma(e)$ is a static criterion, like chord-height tolerance or length-scale, Alg.M can then be re-applied to $C X \mathcal{N}(V)$ to remove any small angles created during edge refinement and swapping.

\subsection{Length-Scale Transport}

Quad/Octrees have been used extensively in unstructured mesh generation both for proximity testing and the establishment of local length scales. $[29,30,31,32,33]$ The Cartesian mesh generation system of Ref.[2] provides just such a mechanism. Through the use of compact unstructured data structures, this particular Cartesian method preserves the ability to directionally refine Cartesian cells. ${ }^{[34]}$ Mesh generation schemes such as this produce geometry-refined meshes with smaller cells near smaller features of the geometry. In addition, since it is an embedded-boundary approach, the Cartesian mesh generator can outputs a set of cut-cells, $C$, which envelope the surface of the geometry. The triangulation output from Alg.M and chord-height refinement provides sufficient input for Cartesian mesh generation.

Figure 6 demonstrates the geometry-adapted Cartesian mesh's ability to transport length-scales between disjoint components. Since our intent is to produce a method which is fully automated, this property is essential.

The set of $N_{C}$ cut-cells, $C$, is inserted into an Alternating Digital Tree (ADT) ${ }^{[35]}$ so that any edge, $e$, in $C X \mathcal{N}(V)$ can locate the subset of $C$ which intersect it with a $\log \left(N_{C}\right)$ time bound. The ADT returns a short list of cells, which intersect $e$, $C_{e}=e \cap C$. Since these cells may have been created through anisotropic subdivision, we can compute an average size, $h$, for the intersected cells in each coordinate direction, $j \in\{1,2,3\}$ which defines a vector length scale function for every edge in the manifold triangulation.

$$
h_{j}(e) \equiv \frac{1}{N_{C}} \sum_{k=1}^{N_{C}} h_{k, j}
$$

$h_{j}(e)$ may then be used to compute the normalized excess length, $\hat{l}_{j}$, of the edge in each coordinate direction.

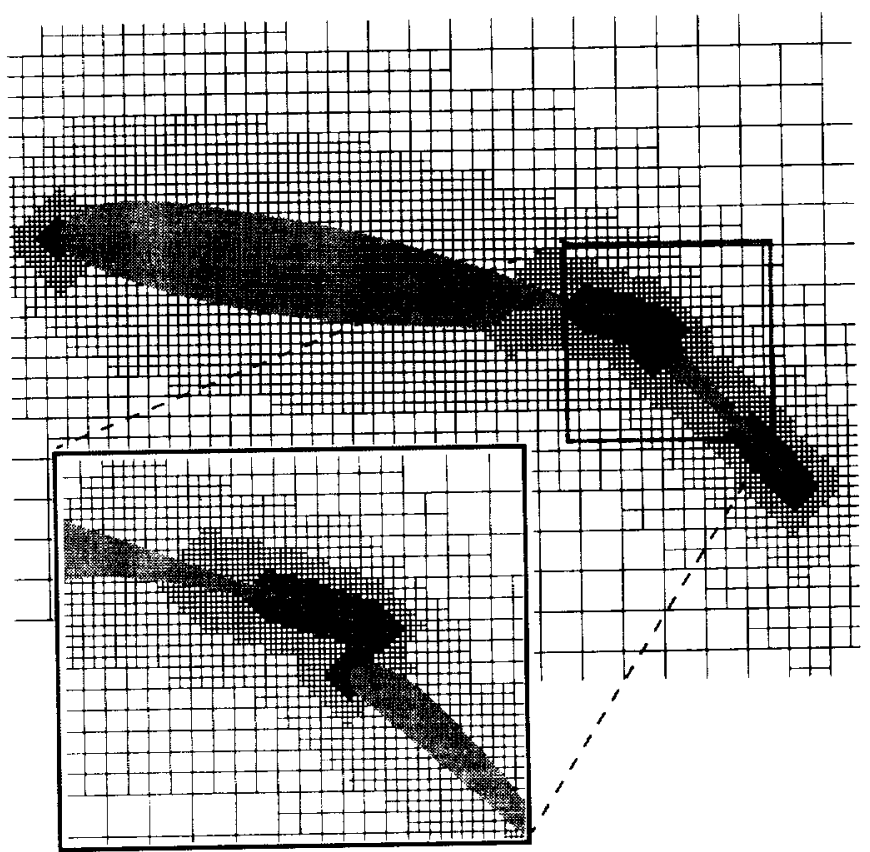

Figure 6. Isotropically refined Cartesian mesh around multi-element airfoil using method of Ref.[2]. Since the mesh is geometrically adapted. it communicates length scales between disjoint geometric entities in close proximity. 

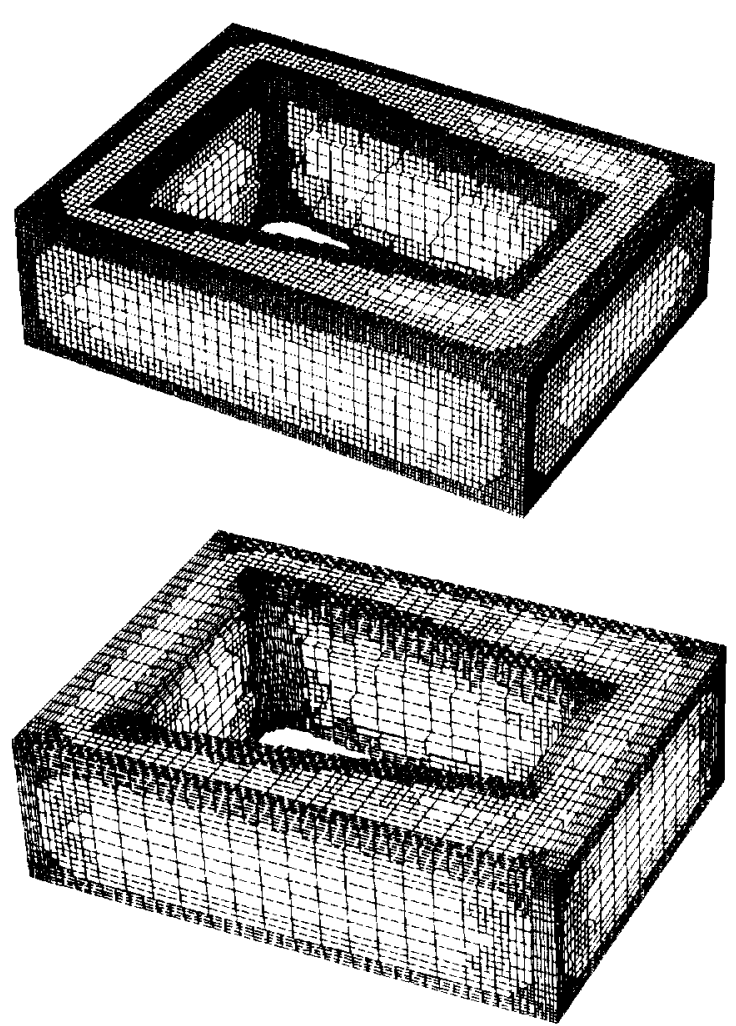

Figure 7. Isotropic (top) and anisotropic (bottom) sets of cutcells from Cartesian mesh generator used to specify length scales for surface triangulation of manufacturing die example.

$$
\begin{gathered}
\hat{l}_{j} \equiv \frac{\left|e_{j}\right|-h_{j}(e)}{h_{j}(e)} \\
l^{*}=\operatorname{Max}\left(\hat{l}_{1}, \hat{l}_{2}, \hat{l}_{3}\right)
\end{gathered}
$$

The scalar $l^{*}$ is computed for each edge and inserted into the priority queue $P Q_{\gamma}$ in Alg.E for length-scale based edge refinement.

Figure 7 displays the set of cut-cells from isotropic and anisotropically refined Cartesian meshes for the manufacturing die example from $\$ 2.2$ (Fig.2). This set of cells defines the vector length scale function for the surface via Eq.(7).

\subsection{An Alternative Insertion Strategy}

The cut-cells in Fig. 7 are interesting in that they suggest an alternative to the incremental mesh improvement algorithm of the preceding sections. Rather than incremental site insertions, one can imagine an approach which simply includes

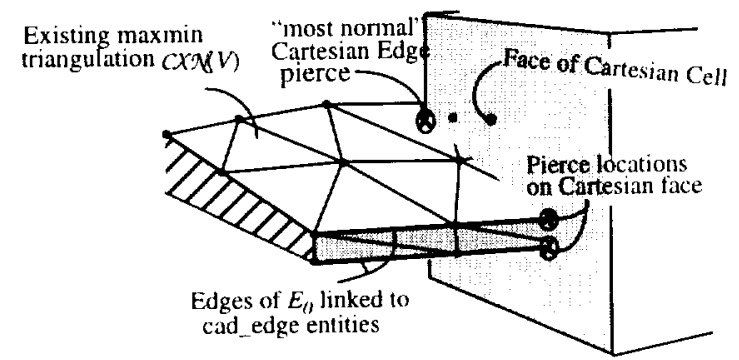

Figure 8. An alternative site insertion strategy based upon pierce locations of the "most normal" edges from cut Cartesian cells and cad_edge/Cartesian cell face pierce points. the intersection points of the "most normal" Cartesian edges with the surface triangulation. In order to preserve the cad_edge features of the geometry, we augment this set with the pierce locations of cad_edges with the faces of the cutcells. Figure 8 demonstrates the construction of a vertex set in this manner.

We explore this alternative using the flap geometry and cutcell set for the flap geometry shown in Figure 9. The surface triangulation formed by enforcing $\tilde{\Phi}_{X N}$ on this point set is shown in Figure 10. In examination of this figure several things are apparent. Despite the fact that the flap is swept, twisted and inclined, the anisotropic Cartesian cell subdivision correctly introduces high-aspect ratio cells along the flap, with vertices clustered at the leading and trailing edges as a result of the geometry adapted Cartesian mesh. Nevertheless, the triangulation is somewhat irregular, both as a result of swaps enforced by the $\tilde{\boldsymbol{\Phi}}_{X N}$ predicate and at locations corresponding to the interfaces between adaptation levels of the cut Cartesian cells. Other triangulation predicates (Minmax, for example) may alleviate some of this irregularity. ${ }^{[36][37]}$

A truly successful implementation of this insertion strategy would require explicit mesh smoothing passes to mitigate these irregularities. In addition, in examining the triangula-
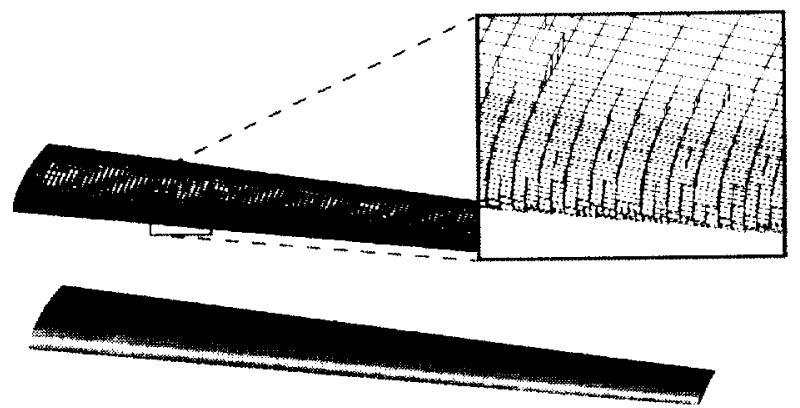

Figure 9. Flap geometry and associated set of anisotropically generated cut Cartesian Cells

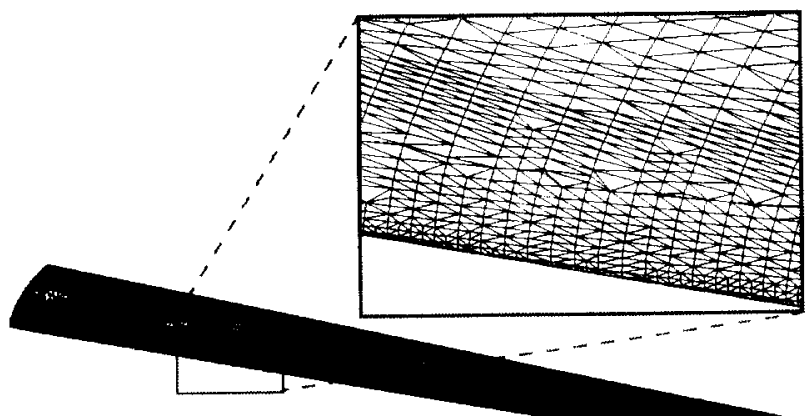

Front view

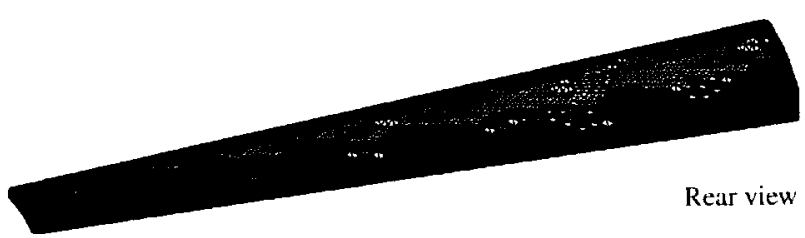

Figure 10. Surface triangulation formed using sites of "most normal" Cartesian edges from Fig.9 and cad_edge pierce locations from Fig.8. 
tion at the leading edge (close-up, Fig. 10) we can discern a region of irregularity associated with the switch over of pierce locations from horizontal Cartesian edge-pierces to vertical edge-pierces. Mesh smoothing would be beneficial in this area as well.

One very attractive property of this approach is that it completely decouples the final triangulation from the description of the geometry. The final vertex set is not a function of the initial vertex set. Therefore, any "history" is removed from the triangulation. The surface triangulation in Fig. 10 should be considered preliminary, and although it has some attractive properties this approach was not aggressively pursued.

\section{Results and Discussion}

This section presents example meshes on several CAD parts from a variety of sources. All the example parts were read in their native CAD file format using the CAPRI API without special treatment. The investigations focus on examination of issues raised in the presentation of the triangulation algorithm in $\$ 3.3$, the edge refinement strategy from $\$ 3.5$ and the length scale transport from $\$ 3.6$.

\subsection{Minimum Angle Bound}

In $\$ 3.3$, Algorithm $\mathrm{M}$ was presented without firm proof of termination. Moreover, the discussion noted that the modified site insertion strategy for obtuse triangles violates one of the assumptions that establishes a bound on the output size of the mesh in the PSLG method. It is therefore necessary to demonstrate the performance of the Alg.M to show that it both terminates and produces meshes with an economy similar to that of the PSLG method upon which it is based.

Figure 11 contains a histogram of the evolution of the smallest angle in the mesh as Alg.M proceeds on the manufactur-

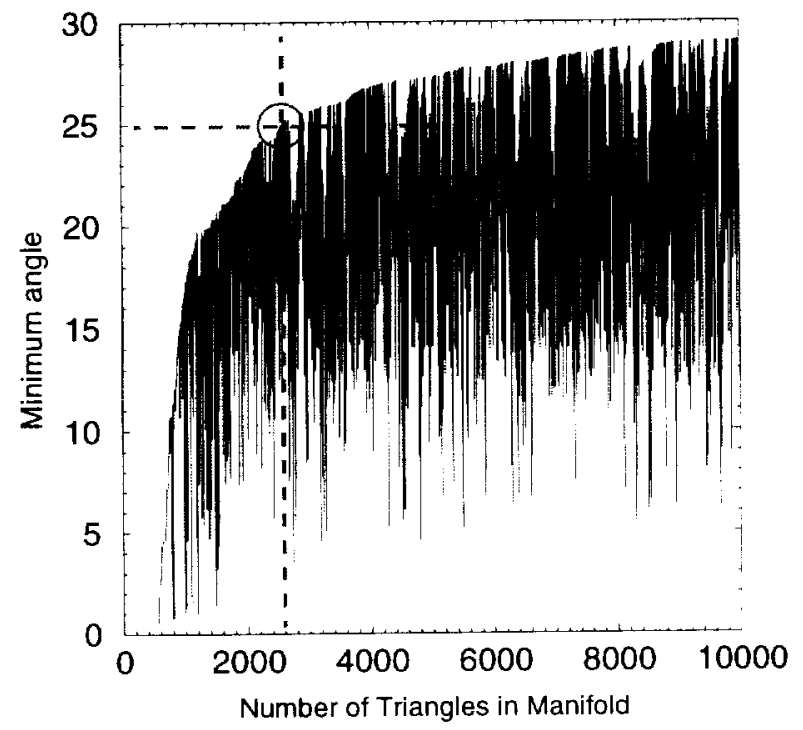

Figure 11. Histogram of minimum angle during mesh evolution using Alg.M (without the edge refinement of $\$ 3.5$ ) for the manufacturing die example presented earlier. In this example, a mesh with a minimum angle of $25^{\circ}$ would contain 2606 triangles.

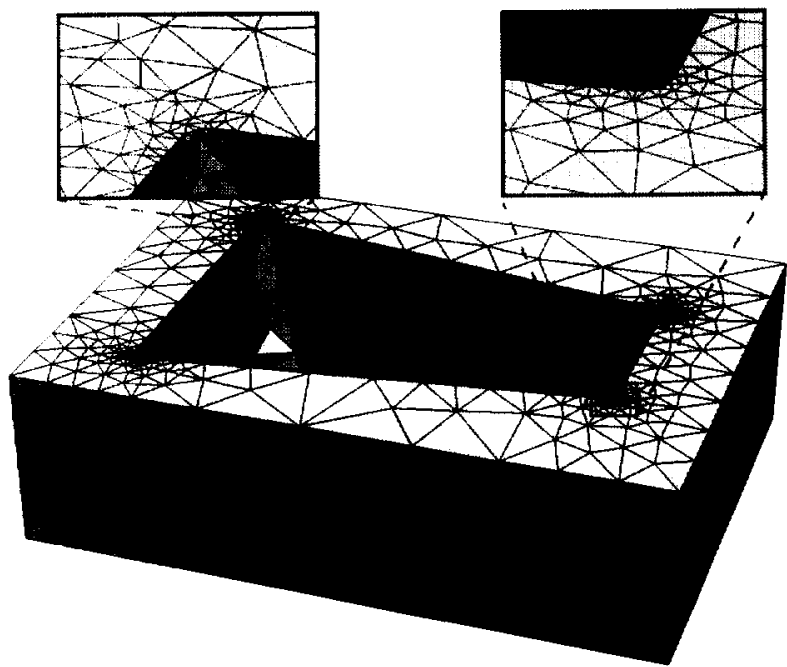

Figure 12. Quality manifold triangulation for manufacturing die example generated with Alg.M. in \$3.3. Mesh improvement terminated after generating 2606 triangles when the minimum angle in the triangulation reached $25^{\circ}$. Chord-height and length-scale refinement not used.

ing die example problem used in earlier illustrations. While this curve is far from monotone, it clearly displays the steady improvement of the minimum angle in the mesh as the site algorithm proceeds. The steep initial rise indicates rapid annihilation of extremely small angles in the mesh, and the mesh achieves a minimum angle of almost $29^{\circ}$ by the end of the histogram.

The dashed line at $25^{\circ}$ highlights the first time that all angles in the mesh exceeded this value. Tracing this value on the abscissa shows that setting the angle bound, $\alpha$, to $25^{\circ}$ will cause Alg.M to terminate after generating 2606 triangles. Figure 12 shows the resulting triangulation. As discussed in $\$ 3.1$ and $\$ 3.3$, the presence of an angle bound ensures that small features are surrounded by proportionally small triangles (see the inset frames in Fig. 12), and that the mesh length scale varies smoothly over the part. The smallest angle in the mesh is $25.02^{\circ}$, which corresponds to a maximum aspect ratio of between 2.36 and 4.7 (by Eq.(3)).

While the histogram in Figure 11 shows a steady increase in the minimum angle, there is an irregular array of downward spikes in the profile. In the presentation of the PSLG algorithm, Ref.23 included a similar histogram, and noted the same characteristic. Consider the two triangles $t_{1}$ and $t_{2}$ shown at the left in Figure 13, Assume that $\theta_{1}$ is the smallest

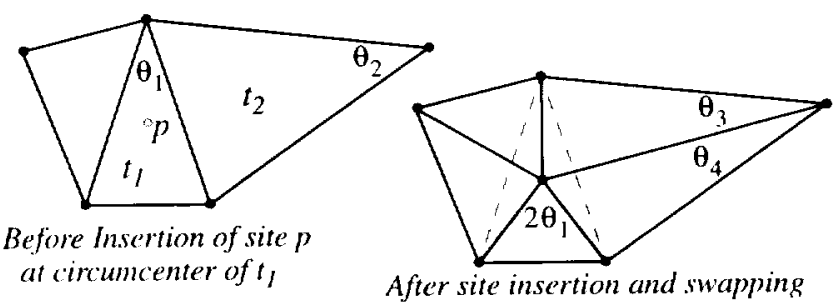

Figure 13. Mechanism responsible for downward spikes in histogram of minimum angle as Alg.M proceeds. If $\theta_{1}$ is initially the smallest angle in the mesh, angles $\theta_{3}$ and $\theta_{4}$ may be smaller after insertion of $p$ and enforcement of $\Phi_{X N}$ by edge-swapping. 
angle in the mesh lying in triangle $t_{l}$. Furthermore, assume that $t_{1}$ 's face neighbor, $t_{2}$, has a small angle $\theta_{2}$ opposite the shared edge which is only slightly larger than $\theta_{1}$. Site $p$ will get added at $t_{l}$ 's circumcenter improving $\theta_{1}$ to $2 \theta_{1}$. After application of the maxmin predicate $\tilde{\Phi}_{X N}$ on the shared edge, the swapped configuration at the right of Fig. 13 may occur. This configuration includes two new triangles with a minimum angles $\theta_{3}$ and $\theta_{4}$ either of which may now be the smallest angle in the mesh and may actually be smaller than the original angle $\theta_{1}$.

With this behavior understood, Figures 14 and 15 present angle histograms and example meshes for a more complicated set of parts. Alg.M was run on CAD parts for the main
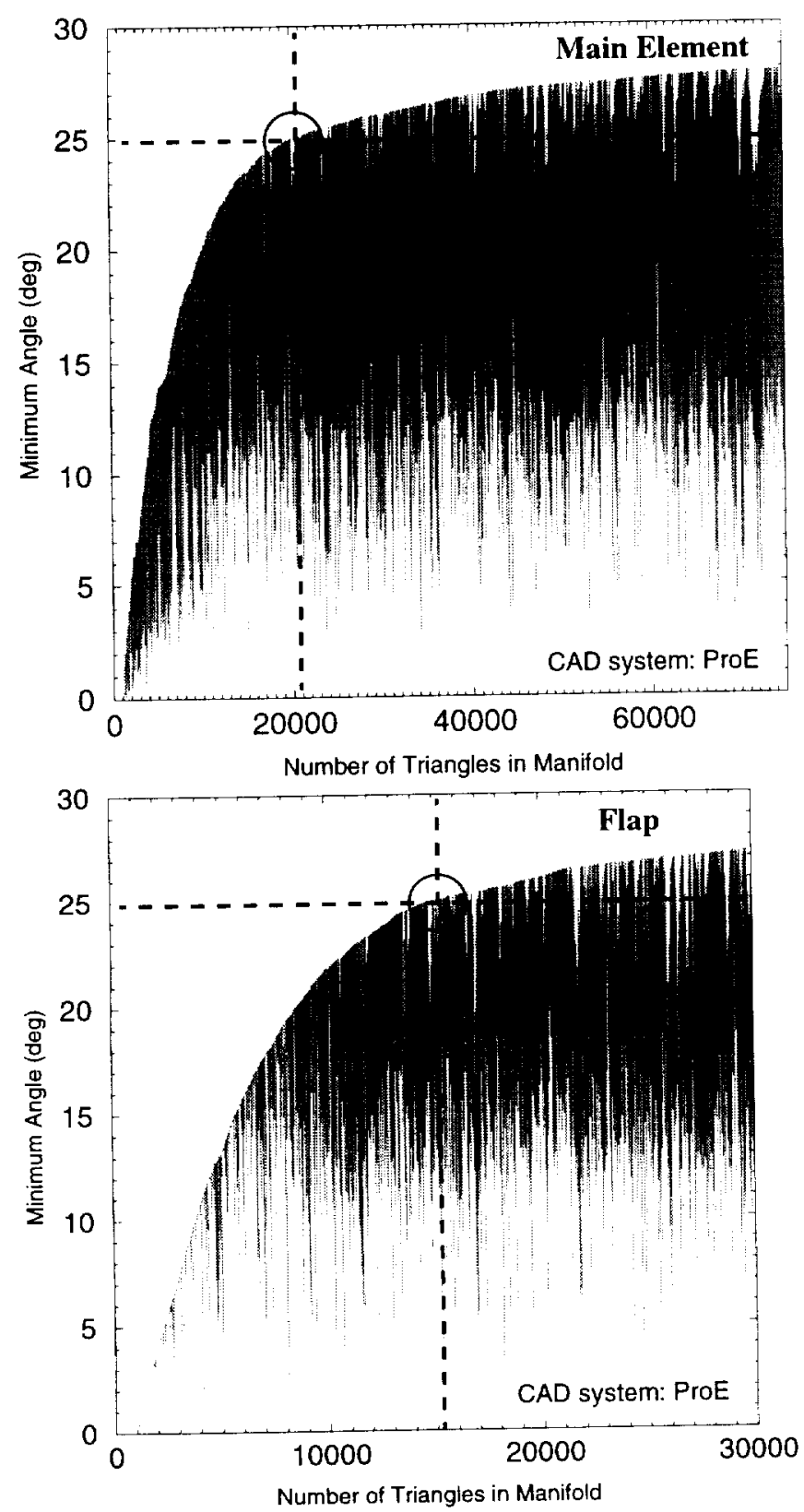

Figure 14. Angle histograms for Alg.M on the main element of a transport wing (upper), and on the main flap (lower). An angle bound of $25^{\circ}$ would produce triangulations with 20846 and 15334 triangles on the main element and flap respectively.

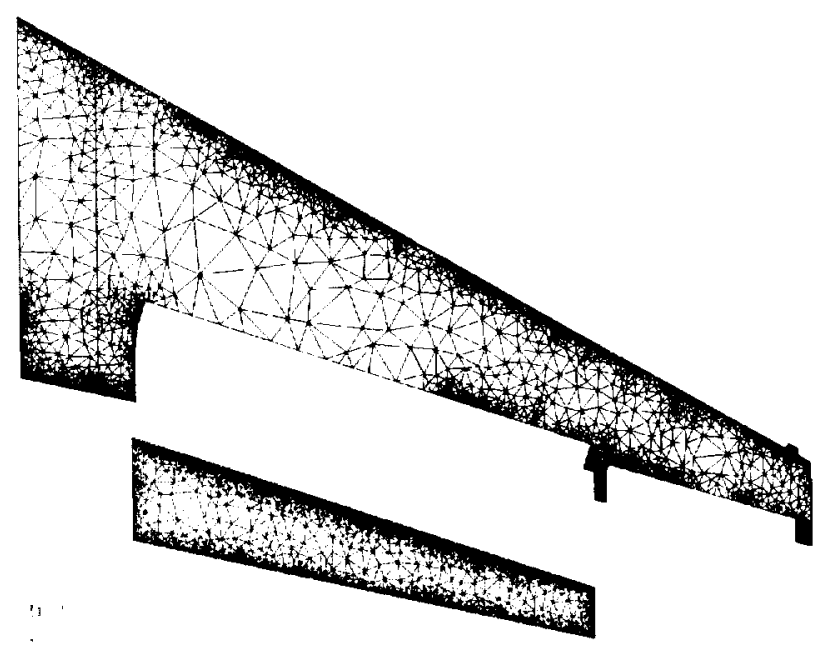

Figure 15. Bounded angle triangulations of main element of a transport aircraft wing and flap generated by Alg.M in $\$ 3.3$. Minimum angle $25^{\circ}, 20846$ triangles on wing, 15334 triangles on flap.

element of a transport wing, and a flap element for the same wing. The main element consisted of 224 rational B-spline curves and 36 trimmed NURBS surfaces. The flap contained 31 rational B-spline curves and 10 trimmed NURBS surfaces.

The crosshairs on the curves in Fig. 14 show that with a $25^{\circ}$ angle bound, Alg. M will produce 20846 triangles on the main element and 15334 triangles on the flap. Figure 15 displays these triangulations.

The histograms in Figure 14 bear close resemblance to the one presented for the simple die example shown in Fig. 11. All of these profiles are characterized by a sharp initial angle improvement and then a rolling-off as the minimum angle climbs above about $20^{\circ}$. All three profiles exceed $27^{\circ}$, but reaching $30^{\circ}$ seems unlikely.

The abscissa values in Fig. 14 indicate relatively large triangulations as compared with the die example. The size-optimality property cited in $\$ 3.1$ and $\$ 3.3$ implies that triangle size must vary smoothly between different sized constraints. In both of these examples the smallest constraint in the capri triangulation was a factor of $10^{4}$ smaller than the largest constraining edge. In addition, practical experience with the algorithm indicates that larger initial triangulations, $S_{C}$, (Eq.(2)) have a proportionally slower initial rise in their angle profiles. This seems reasonable since if the CAPRI triangulation is complex there may initially be many bad angles which need improvement. Often CAD parts are unnecessarily complex for reasons dating to the specific events in their creation. Our experience with $\mathrm{CAD}$ repair software indicates that CAPRI generally produces less complex (sometimes by an order of magnitude) initial triangulations if the parts have been processed by CAD repair tools. Since tolerance to poor CAD parts is one type of robustness that we seek in this research, neither part shown in Fig. 15 underwent such repair prior to creation of these triangulations. 


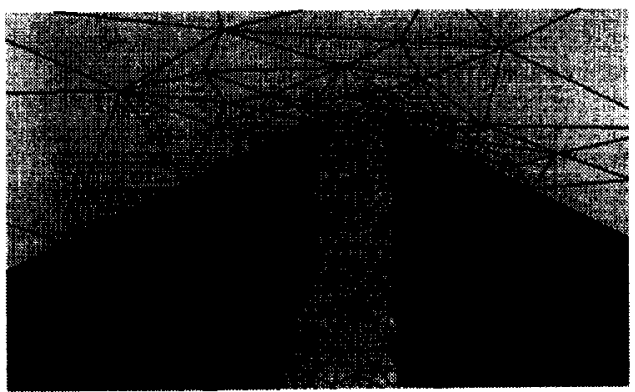

Figure 16. Interior corner of manufacturing die example triangulation after imposition of a chord-height tolerance of $1 . \times 10^{-4}$ normalized by the maximum outer dimension of the part. Edge refinement was performed using Alg.E. in $\$ 3.5$

With the minimum mesh angle restricted to $25^{\circ}$, triangles are again restricted to aspect ratio's less than 4.7 . Such an isotropic mesh is very inefficient at meshing features with curvature in only one dimension. Therefore, if one intends to produce meshes for viscous computation, a substantially smaller angle bound may be appropriate.

\subsection{Chord-Height Refinement}

Enforcement of the angle bound does not guarantee that the edges are refined when they are sufficiently far from the underlying surface. Alg.E in $\$ 3.5$ presented an edge refinement strategy for automatically breaking edges whose midpoints which are far from the geometry. Figure 16 shows an interior corner of the die example after the imposition of a chord-height tolerance of $10^{-4}$ times $L$, where $L$ is the maximum outer dimension of the part. Away from the curved interior corners, the triangulation remains unchanged since the faces of the die are planar.

\subsection{Length Scale Refinement}

Once the initial triangulations of Figs. 12 and 15 have been supplemented with the addition of sites to match the chordheight bound, the triangulations accurately describe the geometry of the part to be meshed. These triangulations then need refinement to reflect the length-scales brought in by the cut Cartesian cells.

The set of anisotropically refined cut-cells from Fig. 7 was used to define a vector length scale function for each edge in the triangulation using eq.(7). Edges were processed with Alg.E using a priority queue based on the maximum component of the normalized excess length function from eqs.(8) and (9), $P Q_{l^{*}}$. Figure 17 displays the resulting length-scale adapted mesh for the die example. This manifold triangulation shown contains 17384 triangles and processing of the $P Q_{l^{*}}$ was halted after achieving a value of $l^{*}=1.0$. In addition to the $l^{*}$, based edge refinement, the minimum angle bound was set to $15^{\circ}$ and a chord-height tolerance of $1 \times 10^{-4} \mathrm{~L}$. In comparing this triangulation with its earlier forms (in Figs. 12 and 16) one sees substantial refinement of the triangulation in response to the length-scale information. However, such a comparison also shows that application of

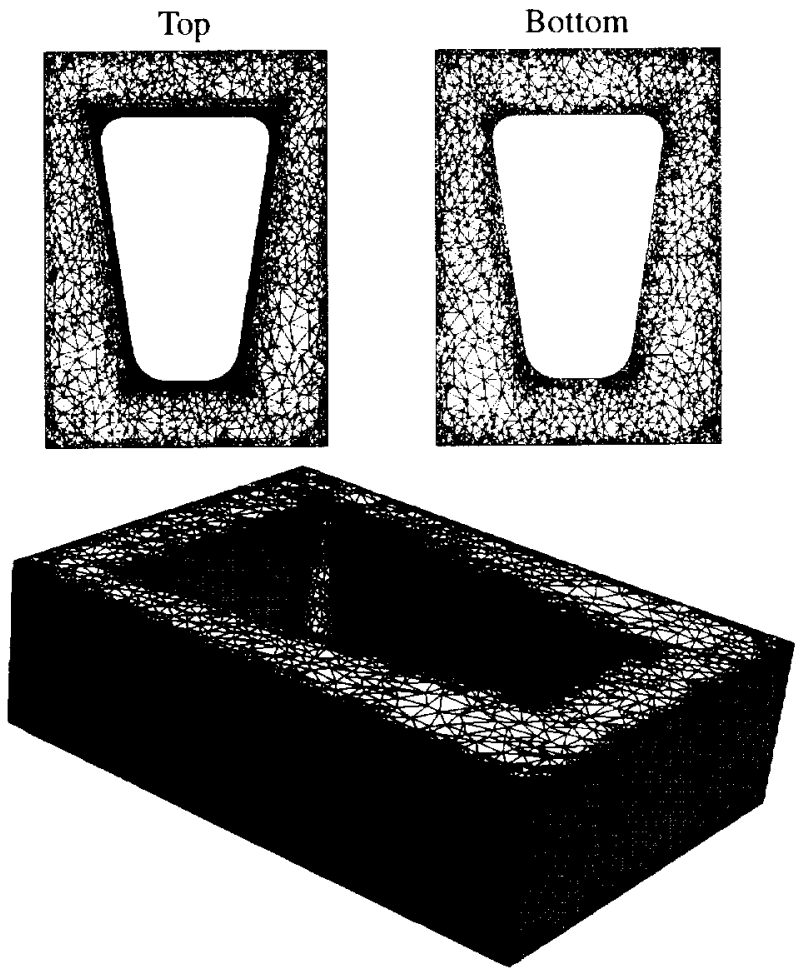

Figure 17. Top, bottom and 3-view of manufacturing die example geometry after length-scale refinement using the anisotropic Cartesian cut-cells from Fig.7. 17384 triangles, $\alpha=15^{\circ}$, maximum chord-height set to $1 . \times 10^{-4} \mathrm{~L}$.

Alg.E has apparently failed to convey the anisotropy of the cut-cells to the final triangulation (c.f. Fig.7). The reasons behind this shortcoming are obvious.

(1) Our edge-refinement uses the very simplistic strategy of simply breaking edges at their midpoint, without consulting $\bar{h}(e)$ for information regarding desired anisotropy prior to point placement.

(2) The triangulation predicate $\tilde{\Phi}_{X N}$ fights anisotropy after every insertion, since it forces edge swaps in order to maximize the minimum angle.

In order to use this approach for creating acceptable stretched triangulations, then the triangulation predicate and point placement strategies need to be modified. One interesting approach would be to modify $\tilde{\boldsymbol{\Phi}}_{X N}$ with $\bar{h}(e)$ by applying a scaling during evaluation. In this way, the edge-swapping will be cognizant of the local stretching information. Such an approach is conceptually quite similar to the locally-mapped Delaunay methods sometimes used in 2-D. ${ }^{[38]}$ Ref.[39] examined the performance of alternative triangulation predicates combined with modified site insertion rules and demonstrated that they can be effective in generating smooth stretched triangulations. Both of these approaches merit investigation since they fit well within the framework of the present algorithm, especially since the stretching information in $\bar{h}(c)$ is currently not utilized.

Figure 18 contains length-scale adapted triangulations for the main wing element and flap geometries shown earlier in 


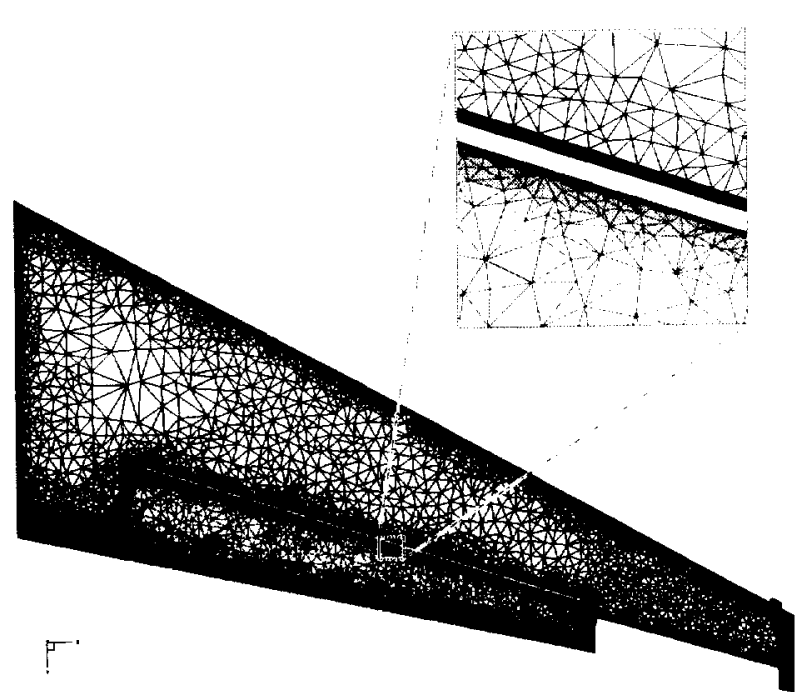

Figure 18. Transport aircraft wing and flap geometries from Fig. 15 after length-scale matching as described in \$3.6. The wing and flap have 34720 and 26640 triangles respectively. Inset frame shows matching length-scales at the flap gap. Minimum angle $15.03^{\circ}$. Chord-height tolerance set to $1 \times 10^{-4}$ of the span.

Fig.15. These triangulations were again created using a minimum angle bound set to $15^{\circ}$ which resulted in a smallest angle of $15.03^{\circ}$. A chord-height tolerance of $1 \times 10^{-4} \mathrm{~L}$, was used, however, this value is somewhat lax for a serious computation. Length-scale refinement was stopped at $l^{*}=1.0$. The triangulation of the main element has 34720 triangles while the flap has 26640 . In comparing these triangulations to those in Fig. 15 notice that the flap leading-edge length scales have been transported through the flap gap to the flat surfaces of the main element. The small frame inset in Fig. 18 is included to show this detail.

\section{Conclusions and Future Work}

\subsection{Conclusions}

This work focused on the direct use of CAD geometry for the automatic generation of closed manifold surface triangulations. Our approach used the CAPRI API to provide a modeler independent method of interacting directly with the CAD system's geometry engine. This approach avoids data translation which can deplete a model of topological information, and avoids the consistency conflicts which can occur when different geometry engines attempt to infer topology from a parts geometry. In addition, it permits the generation of new vertices using the same constructors which were used to create nearby geometry already in the part, thus avoiding building an inconsistent model.

A second novel aspect of this work was the development of a new curved surface meshing technique based upon the use of approximate circumcenters for site insertion. The method maintains a locally maxmin triangulation and produces a bounded aspect ratio manifold triangulation. The algorithm was demonstrated on CAD parts of varying complexity and reliably produced triangulations with minimum angles in excess of $27^{\circ}$.
An edge refinement algorithm was also presented which was used to ensure that edges in the triangulation match the underlying part model to within a specified tolerance. Edge refinement was also used in conjunction with an anisotropically refined Cartesian mesh to ensure that triangles on disjoint surfaces in close proximity maintain matching lengthscales.

An alternative site insertion strategy was examined in which the final vertex set was taken from the "most normal" pierce points of a geometry-adapted Cartesian mesh with an early triangulation. This approach remains attractive since it fully decouples the final triangulation from any intermediate models used to initially describe the surface.

\subsection{Future Research}

- Despite the fact that the cut Cartesian cells define a vector length scale function in Eq.(8), our simple (edge subdivision) insertion strategy does not make use of this information. Since the adapted Cartesian mesh was subdivided in response to surface curvature, $h_{j}(e)$ should be able to provide more guidance concerning the placement of new sites. Since we intend to produce surface triangulations that include high aspect ratio triangles finer control of site placement must is a necessity. Two strategies for including this information were cited in $\$ 4.3$, as well as alternative triangulation predicates.

- Although Alg.M. produces meshes with generally smooth length-scale variation. There is occasionally a discernible irregularity in the triangulations. This behavior becomes more pronounced when higher angle bounds are specified. Similar behavior has been noted in the PSLG algorithm [23], although in 2-D the behavior seems less pronounced. One possible source for this is the abrupt change in insertion location from circumcenter to centroid (of $t_{o p p}$ ) if an obtuse triangle is encountered. Alternative strategies should be investigated since this behavior can degrade the efficiency of the triangulations.

- The initial triangulation returned by CAPRI depends strongly upon the "history" of the CAD part. Such effects are evident in the triangulation of the main wing element in Fig. 15, for example, where the outboard portion of the flap cut-out is excessively resolved due to relics of the part's creation process. The only effective strategy we've found for avoiding this is to "clean" the geometry using CAD repair software. Alternative approaches should he investigated.

\section{Acknowledgments}

The CAPRI API was developed by R. Haimes under NASA Lewis Contract: NAG3-2019. The authors wish to thank contract monitor G. Follen for his support of this work. The authors also thank J. Ruppert of Axys Pharmaceuticals and T. Pulliam, Y. Liu and P. Walsh at NASA Ames for many productive and insightful discussions over the course of this work. 


\section{References}

[1] Cosner, R. "Issues in aerospace applications of CFD analysis," AIAA Paper 94-0464, Jan 1994.

[2] Aftosmis, M.J., Berger, M.J., Melton, J.E., "Robust and efficient Cartesian mesh generation for component-based geometry." AIAA Paper 97-0196, Jan 1997.

[3] Löhner.R., "Finite element methods in CFD: Grid generation, adaptivity and parallelization." AGARD Special course on unstructured Grid methods for Advection dominated Flows, AGARD-R-787, May, 1992.

[4] Marcum, D.L., and Weatherhill, N.P., "Unstructured grid generation using iterative point insertion and local reconstruction." AIAA Jol. 33(9), Sep. 1995.

[5] Pirzadeh, S., "Unstructured viscous grid generation by the advancing layers method." AIAA Paper 93-3453-CP. Jun. 1993.

[6] Wang, Z.J., Przekwas, A., and Hufford, G., "Adaptive Cartesian/adaptive prism grid generation for complex geometry," AIAA Paper 97. 0860, Jan. 1997.

[7] ICEM CFD/CAE V.3.1.3: User Manual, Volume 2, Unstructured Grid Generation. ICEM Systems Inc. Berkeley CA, Nov. 1994.

[8] Bohn J.W., and Zozny, M.J., "Automatic CADmodel repair: Shell-closure." Proc. Symp. On Freeform Fabrication. Dept. of Mech. Eng., Univ. of Texas at Austin, 1992.

[9] Guéziec, A., Taubin, G., Lazarus, F., and Horn, W. "Cutting and stitching: Efficient conversion of a non-manifold polygonal surface to a manifold.", IBM-RC-20935. IBM Research Division, Yorktown Heights, NY, Jul. 1997.

[10] "VGRID: An unstructured tetrahedral grid generator based on the advancing front method." Vigyan Inc. Hampton VA. http:// www.vigyan.com

[11] “CFD-GEOM Users Manual," CFD Research Corp., Huntsville Al, 1997.

[12] Reed, K. "The Initial Graphics Exchange Specification (IGES) Version 5.1", Sept. 1991.

[13] "Industrial automation systems and integration -- Product data representation and exchange -Part 1:Overview and fundamental principles." ISO /TR 10303-1. International Standards Org. Genève, Switzerland, 1994.

[14] 3D Systems Inc. Stereolithography Interface Format Specification. 1988.

[15] Haimes, R., "CAPRI: Computational Analysis Programming Interface A Geometry Based Infra-Structure for Engineering Analysis and Design." Dept. of Aero and Astro Eng. Mass. Inst. of Tech. Cambridge, Ma. 1997.

[16] Yap, C., "Robust geometric computation." CRC Handbook of Discrete and Computational Geometry Eds. Goodman J.E., and O'Rourke, J., CRC Press, Boca Raton Fl., 1997.

[17] Shewchuk, J.R., "Adaptive Precision FloatingPoint Arithmetic and Fast Robust Geometric
Predicates."CMU-CS-96-140. Carnegie Mellon Univ. School of Computer Science, May, 1996.

[18] Farin, G., Curves and Surfaces for Computer Aided Geometry Design, A Practical Guide, Academic Press, 1990.

[19] Michelucci, D. “The Robustness Issue." Internal report, Laboratoire d'Image de Synthèse de St Etienne, France. See http://www.emse.fr/ $\sim$ micheluc/english/michelucci.html

[20] Goldberg, D., "What every computer scientist should know about floating-point arithmetic." ACM Comput. Surveys, 23(1):5-48, Mar. 1991.

[21] Bern, M., and Eppstein, D., "Mesh generation and optimal triangulation." in Computing in Euclidean Geometry, Du and Hwang eds., World Scientific, 1992.

[22] Chew, L.P., "Guaranteed-quality triangular meshes." Technical Report, TR-89-983. Computer Science Dept, Cornell Univ. Apr. 1989.

[23] Ruppert, J., "A Delaunay refinement algorithm for quality 2-dimensional mesh generation." Jol. of Algorithms, 18(3):548-585, May 1995.

[24] Chew, L.P., "Guaranteed-quality mesh generation for curved surfaces." Proc. of the Ninth Annual Symposium on Computational Geometry, 274-280. ACM, 1993.

[25] Green, P.J., and Sibson, R., "Computing the Dirichlet tessellation in the plane." The Computer Journal, 2(21):168-173, 1977.

[26] Real/Expr homepage, 1996. Source code, documentation, examples and literature. http://simulation.nyu.edu/projects/exact.

[27] Ouchi, K., "Real/Expr: Implementation of an exact computation package", Master's Thesis, New York Univ., Dept. of Comp. Sci., Courant Inst. NY. Jan 1997.

[28] Trimaran homepage, 1998. http://reactilp.nyu.edu.

[29] Yerry, M.A., Shepard, M.S., "Automatic threedimensional mesh generation by the modifiedoctree technique." Internat. Jol. Num. Meth. Eng. 20:1965-1990, 1984.

[30] Pirzadeh, S., "Structured background grids for generation of unstructured grids by advancing front method." AIAA Paper 91-3233. Sep. 1991.

[31] Mavriplis, D.J., "An advancing front Delaunay triangulation algorithm designed for robustness." AIAA Paper 93-0617, Jan. 1993.

[32] Löhner.R., "Matching semi-structured and unstructured grids for Navier-Stokes calculation." ALAA Paper 93-3348, Jun. 1993.

[33] Khawaja A., McMoris, H., and Kallinderis, Y., "Hybrid grids for viscous flows around complex 3-D geometries including multiple bodies." AIAA Paper 95-1685. Jun 1995.

[34] Berger, M.J., and Aftosmis, M.J., "Aspects (and aspect ratios) of Cartesian Mesh Methods." Lecture Notes in Physics: Proc. of the 16th Internat. Conf. on Num. Meth. in Fluid Dynam., Springer-Verlag, Heidelberg, 1998.

[35] Bonet, J., and Peraire, J., "An Alternating Digital Tree (ADT) algorithm for geometric search- 
ing and intersection problems." Int. Jol. Num. Meth. Eng., 31:1-17, 1991.

[36] Marcum, D.L., "Generation of unstructured grids for viscous flow applications." AIAA-Paper 95-0212, Jan. 1995.

[37] Barth, T.J., "Numerical aspects of computing viscous high Reynolds number folow $s$ on unstructured meshes." AIAA Paper 91-0721, Jan. 1991.

[38] Mavriplis, D.J., "Adaptive mesh generation for viscous flows using Delaunay triangulation." Jol. of Comp. Physics, 90(2):271-291, 1990.

[39] Barth, T.J., "Steiner triangulation for isotropic and stretched elements." AIAA Paper 95-0213, Jan. 1995. 